\title{
ESTUDO DA MODELAGEM DO AMORTECEDOR MAGNETO-REOLÓGICO
}

\author{
Alexandre Silva de Lima \\ Doutor em Engenharia Mecânica pela Universidade Federal do Rio de Janeiro (COPPE- \\ UFRJ), \\ Rio de Janeiro, RJ, Brasil. \\ alexandre.silva.lima@gmail.com
}

Fernando Augusto de Noronha Castro Pinto, Dott-IngDoutor em Engenharia Mecânica pela Techische Universität Hamburg Harburg, Hamburgo, Alemanha. fcpinto@ufrj.br

\section{RESUMO}

Os fluidos magneto-reológicos (MR) são denominados materiais inteligentes, pois, a exposição a um campo magnético influência diretamente na viscosidade, elasticidade e plasticidade do fluido. Quando não exposto a um campo magnético, o fluido se comporta como um fluido newtoniano comum, porém quando exposto, o fluido apresenta um comportamento visco-plástico cuja tensão de escoamento é função da intensidade do campo magnético. A aplicação mais comum dos fluidos MRs encontrase nos amortecedores magneto-reológicos. A capacidade de os fluidos MRs alterar de maneira reversível a sua viscosidade, trazem aos amortecedores MRs uma grande potencialidade em aplicações de controle e isolamento de vibrações. Os amortecedores MRs são aplicados em diversas áreas como: realimentação tátil, suspensão automotiva, assentos de veículos pesados, amortecedores sísmicos e em próteses trans-femorais. O objetivo deste trabalho é apresentar a modelagem matemática capaz de descrever o comportamento dinâmico não-linear dos amortecedores MRs considerando os modelos paramétricos encontrados na literatura: o modelo de Bingham, o modelo de Gamota e Filisko, o modelo de Bouc-Wen e o modelo de Bouc-Wen modificado.

Palavras-chave: Fluido magneto-reológico. Amortecedor magneto-reológico. Modelagem matemática.

\section{STUDY OF MODEL OF MAGNETORHEOLOGICAL DAMPER}

\begin{abstract}
Magnetorheological fluids are classified as intelligent materials because their rheological properties (viscosity, elasticity and plasticity) changes when they are submitted to magnetic field variations. Those fluids, when not submitted to magnetic fields, have a behavior very near to the Newtonian fluid, and when submitted present a plastic-viscous
\end{abstract}


behavior that depends on the intensity of magnetic field to which they are exposed. The most common application of those fluids is in dampers and breaks because of their ability to change their viscosity in a reversible way. Handling this characteristic together with the controllability of such reversible changes, those devices have been widely investigated as elements of vibration control and isolations systems. But, another plausible application is their use as semi-active actuator. The Magnetorheological damper are applying in some areas like: automotive suspension, heavy vehicle seats, seismic shock absorbers and ak prosthesis. The aim of this work is to present the mathematical model able to describe the nonlinear behavior of a magnetorheological damper, considering some models, as well as Bingham, Gamota e Filisko, Bouc-Wen and Modified Bouc-Wen.

Keywords: Magnetorheological fluids. Magnetorheological damper. Mathematica modeling.

\section{INTRODUÇÃO}

Os fluidos controláveis, também denominados como inteligentes, são aqueles sensíveis ou adaptáveis a alterações nas condições operacionais, visando, assim, a manter o comportamento requerido do sistema.

Os fluidos controláveis apresentam uma ou mais propriedades mecânicas que, devido à influência da variação de alguma grandeza física à qual estejam submetidos como temperatura, campo elétrico, pressão, campo magnético, entre outras, sofram alterações reversíveis (YANG et al., 2002).

Entre os fluidos controláveis os empregados em dispositivos de controle são: os fluidos magneto-reológicos e os fluidos eletro-reológicos (STEFFEN et al., 2004, PONS, 2005, BANKS et al., 1996).

\section{FLUIDOS MAGNETO-REOLÓGICOS}

Os Fluidos Magneto-reológicos (MRs) já existem há aproximadamente vinte anos (YANG et al., 2002). Contudo, somente agora eles têm características estáveis atrativas para aplicação prática, como, por exemplo, elevado campo de tensão e baixa viscosidade. Eles são adequados em certas aplicações como em: suspensões automotivas, amortecimento em assentos de veículos pesados, sistemas de amortecimento sísmicos, próteses inteligentes, e sistemas de realimentação tátil.

Os fluidos magneto-reológicos (MRs) são fluidos que apresentam uma alteração 
reversível em suas propriedades reológicas: viscosidade, elasticidade e plasticidade, quando expostos a um campo magnético. Quando não há a exposição a um campo magnético, o fluido se comporta como um fluido newtoniano comum. Na presença de um campo magnético, o fluido MR apresenta um comportamento visco-plástico com a tensão de escoamento em função da intensidade do campo magnético.

Os fluidos MR são constituídos de micro partículas magneticamente polarizadas (óxido de ferro), suspensas em um fluido excipiente, como por exemplo: óleo mineral, óleo sintético ou silicone.

Quando expostas a um campo magnético, as partículas do fluido apresentam um momento de dipolo paralelo à direção das linhas de fluxo do campo magnético. Desta forma, as partículas, anteriormente dispersas, se alinham ao longo das linhas de fluxo do campo magnético, formando uma estrutura em forma de correntes, conforme é mostrado nos esquemas da Figura 1 (STUTZ, 2005).

Figura 1 - a) Fluido magneto-reológico na ausência de um campo magnético, b) Fluido magneto-reológico na presença de um campo magnético.

(a)

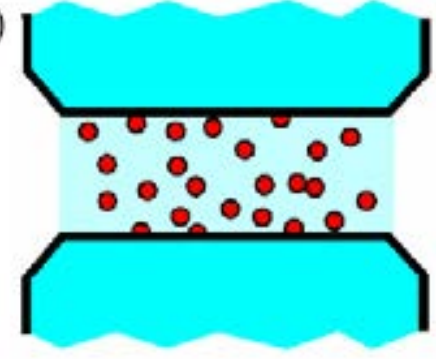

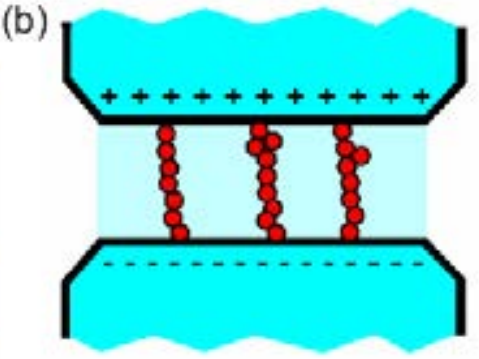

Fonte: (LIMA, 2011).

\subsection{Amortecedores Magneto-Reológicos}

A aplicação mais comum dos fluidos magneto-reológicos são nos amortecedores onde a capacidade destes alteraram de maneira reversível a sua viscosidade fazendo com que os amortecedores tenham uma grande potencialidade em aplicações de controle e isolamento de vibrações.

O esquema de um amortecedor magneto-reológico típico é apresentado na Figura 2. 
Figura 2 - Esquema do amortecedor magneto-reológico.

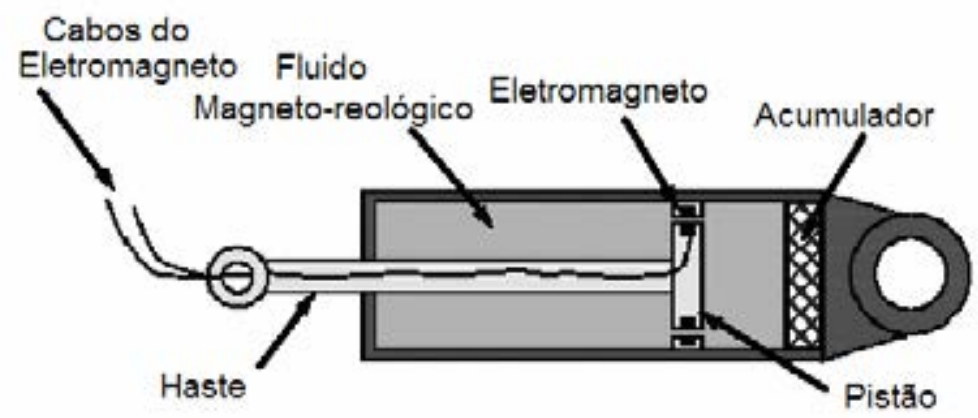

Fonte: (SPENCER et al., 1996).

O fluido magneto-reológico flui através dos orifícios localizados no pistão, quando este está se movimentando. Quando um campo magnético é gerado ocorre um rearranjo das partículas do fluido gerando assim uma certa resistência ao fluxo do fluido. Essa resistência é proporcional ao valor do campo magnético e por conseqüência ao valor da força do amortecedor.

Os amortecedores magneto-reológicos pertencem à classe dos atuadores semiativos.

\subsection{Formulação do Modelo do Amortecedor MR}

Tanto os modelos não paramétricos, (BURTON et al., 1996, EHRGOTT e MASRI, 1992), quanto os paramétricos, (GAMOTA e FILISKO, 1991, SPENCER et al., 1996, KAMATH e WERELEY, 1997, STANWAY et al., 1987), foram considerados para modelar o comportamento observado de amortecedores fluidos controláveis. Os modelos paramétricos, baseados em simples idealizações mecânicas foram considerados por (STANWAY et al., 1985, 1987, GAMOTA e FILISKO, 1991), para descrever o comportamento de fluídos controláveis e dos amortecedores fluidos controláveis.

Existem duas abordagens distintas para o problema da modelagem matemática. Uma é a modelagem do fluido magneto-reológico e a outra é a modelagem do amortecedor magneto-reológico, tendo em vista que o modelo do amortecedor é um modelo fenomenológico, ou seja, é obtido através da observação experimental do comportamento de um sistema mecânico composto por um amortecedor cuja vazão em seu orifício é controlada através do campo magnético. Neste trabalho, o foco da 
modelagem está no amortecedor e, não, no fluido magneto-reológico. A seguir, são descritos os modelos paramétricos:

- Modelo de Bingham

O modelo de Bingham consiste em um elemento de atrito de Coulomb colocado em paralelo com o amortecedor viscoso, como mostrado na Figura 3.

Figura 3 - Modelo de Bingham para amortecedores com fluidos controláveis.

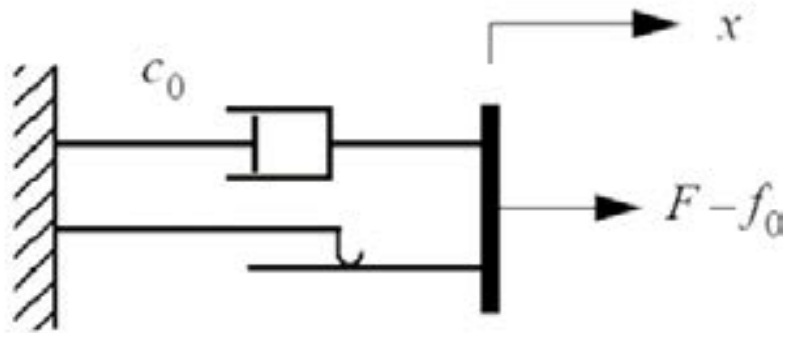

Fonte: (STANWAY et al., 1985, 1987).

Neste modelo, para velocidades do pistão diferentes de zero, a força gerada pelo mecanismo é dado pela Eq. (1):

$$
F=f_{C} \operatorname{sgn}(\&)+c_{0} \&+f_{0}
$$

onde \& é a velocidade relativa das extremidades do amortecedor, sgn( ) é a função sinal, $c_{0}$ é a constante de amortecimento plástico, e $f_{C}$ é a força relacionada à tensão de escoamento do fluido.

Uma parcela de força, $f_{0}$, é incluída para os valores de força medidos devido à presença do acumulador no interior do amortecedor. Com base na Eq. 1, podemos concluir que, se em algum ponto, a velocidade do pistão for igual a zero, a força gerada pelo mecanismo, $\mathrm{F}$, é igual a força de atrito, $f_{C}$.

- Modelo de Gamota e Filisko

GAMOTA e FILISKO, 1991, propuseram uma extensão do modelo de Bingham, o 
qual consiste no modelo de Bingham (elemento de atrito em paralelo com o amortecedor de fluido) em série com um modelo padrão de sólido linear (SHAMES e COZZARELLI, 1992), como é mostrado na Figura 4.

Figura 4 - Modelo proposto por GAMOTA e FILISKO, 1991.

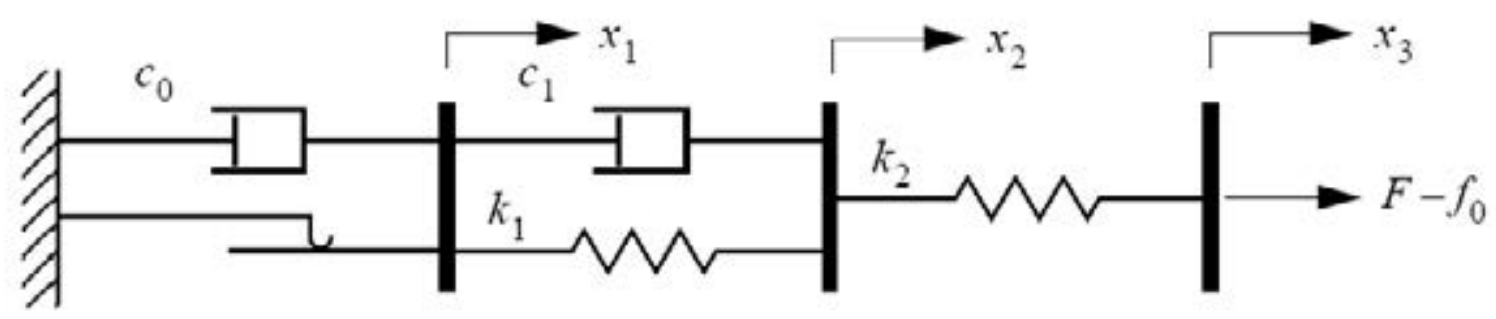

Fonte: (DYKE et al., 1996).

As equações que governam este modelo estão descritas nas Eq. (2) e Eq. (3).

$$
\begin{aligned}
& F=k_{1}\left(x_{2}-x_{1}\right)+c_{1}(\text { \& })+f_{0} \\
& =c_{0} \&+f_{c} \operatorname{sgn}(\&)+f_{0} \quad|F|>f_{c} \\
& =k_{2}\left(x_{3}-x_{2}\right)+f_{0} \\
& F=k_{1}\left(x_{2}-x_{1}\right)+c_{1}+f_{0} \\
& =k_{2}\left(x_{3}-x_{2}\right)+f_{0} \quad|F| \leq f_{c}
\end{aligned}
$$

- Modelo de Bouc-Wen

Um modelo que é numericamente tratável e tem sido usado freqüentemente para modelar sistemas com histerese é o modelo de Bouc-Wen (WEN, 1976).

O modelo de Bouc-Wen é extremamente versátil e pode exibir uma grande variedade de comportamento de histerese.

Um esquema deste modelo é mostrado na Figura 5, onde é formado pela combinação em paralelo de um elemento de histerese de Bouc-Wen (WEN, 1980). 
Figura 5. Modelo de Bouc-Wen.

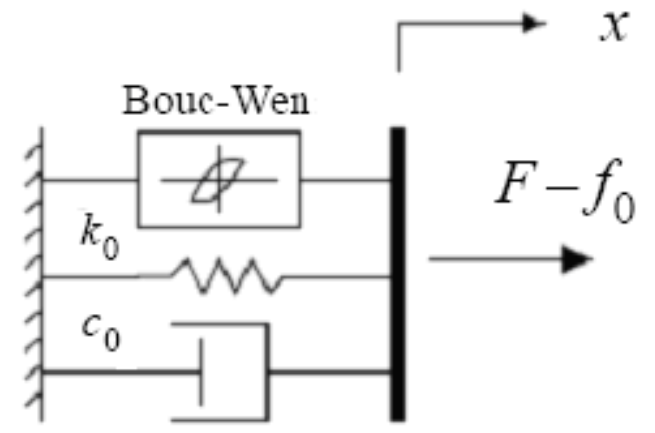

Fonte: (STANWAY et al., 1985).

A força neste sistema é dada pela Eq. (4).

$$
F=c_{0} \&+k_{0} x+\alpha z+f_{0}
$$

onde ${ }^{c_{0}}$ é o coeficiente de amortecimento plástico associado ao modelo de BoucWen, \&é a velocidade relativa das extremidades do amortecedor, $\mathrm{k} 0 \mathrm{e}^{\mathrm{f}_{0}}$ contabilizam o efeito do acumulador, e z é a variável de estado que representa uma variável interna do sistema, chamada de deslocamento de histerese e é descrita pela Eq. (5).

$$
\&=-\left.\gamma|\& z| z\right|^{n-1}-\left.\beta \notin z\right|^{n}+A \&
$$

Pelo ajuste dos parâmetros do modelo, $\gamma, \beta$, A e n, pode-se controlar a linearidade na ausência de carga e a transição suave antes e depois do carregamento e forma da curva de histerese. A força $f_{0}$ devido ao acumulador pode ser diretamente incorporada a este modelo como uma deflexão inicial x 0 da mola linear k0. Aplicando estes valores na Eq. (6) tem-se:

$$
F=c_{0} \&+k_{0}\left(x-x_{0}\right)+\alpha z
$$

(6)

- Modelo de Bouc-Wen Modificado 
Este modelo é formado por um amortecedor $\mathrm{c} 1 \mathrm{em}$ série com o modelo de Bouc-Wen de modo a fornecer o comportamento não linear existente entre a força e a velocidade (SPENCER ET. AL., 1996). Uma mola k1 é adicionada em paralelo com o objetivo de contabilizar a ação do acumulador. É mostrado na Figura 6 um esquema deste modelo.

Figura 6 - Modelo de Bouc-Wen Modificado

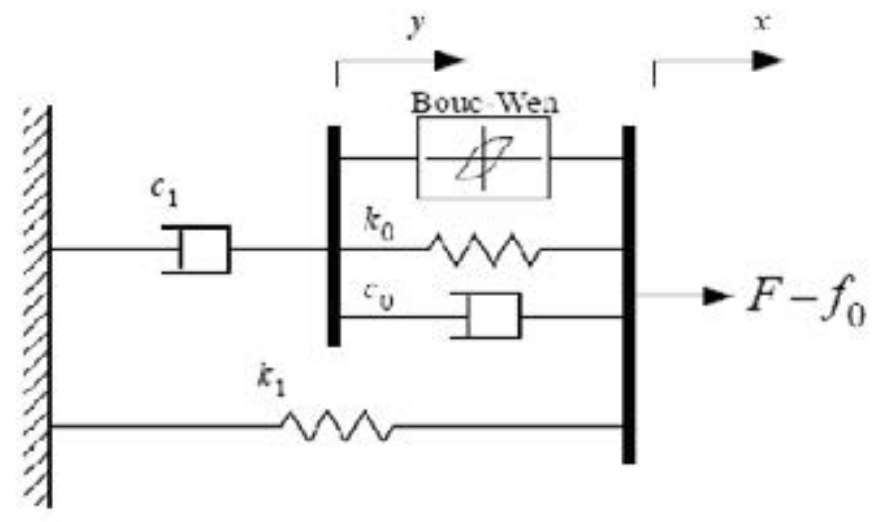

Fonte: (STANWAY et al., 1987).

A força do amortecedor é calculada utilizando a Eq. (7).

$$
F=c_{1} \&+k_{1} x+f_{0}
$$

A velocidade é dada na Eq. (8).

$$
\&=\frac{1}{\left(c_{0}+c_{1}\right)}\left\{\alpha z+c_{0} \&+k_{0}(x-y\}\right.
$$

A variável de estado z é descrita pela Eq. (9).

$$
\&=-\gamma|\&-\not| z|z|^{n-1}-\beta(\&-\beta)|z|^{n}+A(\&-\&)
$$

onde os parâmetros $\gamma, \beta$, A e $\mathrm{n}$ determinam a forma da curva de histerese.

Neste modelo, a rigidez do acumulador é representada por k1 e o amortecimento 
viscoso de amortecimento para grandes velocidades é representado por c0. O amortecedor representado por c1, é incluído para compensar a distorção a baixas velocidades, k0 está presente para controlar a rigidez em altas velocidades, e x0 é o deslocamento inicial da mola k1 associada à força de amortecimento nominal devido ao acumulador.

\section{CONCLUSÃO}

A força determinada pelo modelo de Bingham é descontínua com relação à velocidade, devido à função sinal não ser definida nos pontos de velocidade nula. Portanto, o modelo de Bingham não é suficientemente capaz de descrever a relação entre a força e a velocidade.

O modelo de Gamota e Filisko, por ser uma extensão do modelo de Bingham, ainda não consegue resolver a descontinuidade com relação à velocidade observada no modelo anterior.

O modelo de Bouc-Wen prediz o comportamento força-deslocamento do amortecedor e este possui um comportamento força-velocidade muito parecido com os resultados experimentais (SPENCER et al., 1996). Contudo, foi observado (SPENCER et al., 1996), que ainda existe uma diferença quando em baixas velocidades para a relação força-velocidade.

O modelo de Bouc-Wen modificado melhora a descrição da relação forçavelocidade e atende bem o comportamento do amortecedor na região onde os valores da velocidade são pequenos (SPENCER et al., 1996).

As modelagens mais utilizadas para o amortecedor MR são a de Bouc-Wen e a de Bouc-Wen modificado. Segundo, SPENCER et al., 1996, CARLSON, 1994, o segundo tipo citado representa melhor o comportamento do amortecedor MR.

\section{REFERÊNCIAS}

BANKS, H. T.; SMITH, C.; WANG, Y. Smart Material Structure. Modeling, Estimation and Control, EUA: Wiley-Masson, 1996.

BURTON, S.A.; MARKRIS N.; KONSTANTOPOULOS, I.; ANTSAKLIS, P. J. Modelling the Response of ER Damper: Phenomenology and Emulation, Journal of Engineering 
Mechanics, vol. 122, pp. 897-906, 1996.

EHRGOTT, R.C.; MASRI, S.F. Modelling the Oscillatory Dynamic Behavior of Electrorheological Materials in Shear, Smart Material and Structures, vol. 1, pp. 275285, 1992.

GAMOTA, D.R.; FILISKO, F.E. Dynamic Mechanical Studies of Electrorheological Materials: Moderate Frequencies, Journal of Rheology, Vol. 35, pp. 399-425, 1991.

KAMATH, G.M.; WERELEY, N.M. A Nonlinear Viscoelastic-Plastic Model for Electrorheological Fluids, Smart Materials and Structures, vol. 6, no. 3, pp. 351-358, 1997.

LIMA, A.S., Identificação Experimental de Um Sistema Magneto-Reológico. Tese de Doutorado, COPPE/UFRJ, Rio de Janeiro, Brasil, 2011.

PONS, J. L. Emerging Actuator technologies: A Micromechatronics Approach, England, John Wiley 7 Sons, 2005.

SHAMES, I. H.; COZZARELLI, F. A. Elastic and Inelastic Stress Analysis, Prentice-Hall, 1992.

SPENCER JR., B. F.; DYKE, S. J.; SAIN, M. K.; CARLSON, J. D. Phenomenological Model of a Magnetorheological Damper, ASCE Journal of Engineering Mechanics, v.123, no.3, pp. $230238,1996$.

SPENCER JR., B.F.; DYKE, S.J.; SAIN, M.K. Magnetorheological Dampers: a New Approach to Seismic Protection of Structures, Proc. of Conference on Decision and Control, Kobe, Japan, pp. 676-681, 1996.

STANWAY, R.; SPROSTON, J.L.; STEVENS, N.G. Non-linear Identification of an Electrorheological Vibration Damper, IFAC Identification and System Parameter Estimation, pp. 195-200, 1985. 
. Non-linear Modelling of an Electrorheological Vibration Damper. J. Electrostatics, Vol. 20, pp. 167-184, 1987.

STEFFEN JR., V.; RADE, D.A. Fundamentos e Aplicações de materiais Inteligentes, In: Proceedings of the 3rd Congresso Temático de Dinâmica e Controle da SBMAC, May 31st ? June 3rd, Uberlândia, Brasil, 2004.

STUTZ, L. T. Síntese e Análise de uma Suspensão Semi-Ativa Magneto Reológica Baseada na Abordagem de Controle com Estrutura Variável, Tese de Doutorado, COPPE/UFRJ, Rio de Janeiro, Brasil, 2005.

WEN, Y.K. Method of Random Vibration of Hysteretic Systems, Journal of Engineering Mechanics Division, ASCE, Vol. 102, No. EM2, pp. 249-263, 1976.

WEN, Y.K. Equivalent Linearization for Hysteretic Systems Under Random Excitation, Trans. of the ASME, Journal of Applied Mechanics, vol. 47, pp. 150-153, 1980.

YANG, G.; SPENCER JR., B. F.; CARLSON, J. D.; SAIN, M. K. Large-Scale MR Fluid Dampers: Modelling and Dynamic Performance Considerations, Engineering Structures, vol. 24, pp. 309-323, 2002. 